\title{
Biographie de l'ordinateur R2E-Micral, ou comment faire exister un « micro-ordinateur » dans les années
} 1970s

\section{History of the R2E-Micral computer: how to make a "microcomputer"} exist in the 1970s

\section{Loïc Petitgirard ${ }^{1}$}

${ }^{1}$ Laboratoire HT2S - Cnam, France, loic.petitgirard@lecnam.net

RÉSUMÉ. L'article dresse la biographie d'un micro-ordinateur emblématique, le système Micral, inventé au sein de l'entreprise R2E dans les années 1970. II vise à montrer le jeu des niveaux de culture technique au fil de ce processus d'innovation très complexe : l'omniprésence de la maîtrise technologique, l'importance d'une pensée des usages et la réflexivité des acteurs sur le processus en cours, avec tous les échos au courant "small is beautiful " qui lui est contemporain. L'analyse à ses différents niveaux permet de donner sens à la conception de Micral et ses multiples versions : au cours des années 1970, dans un monde informatique dominé, en termes industriels et en termes de représentations collectives, par la " grande » informatique, Micral est inventé et promulgue le concept même de " microordinateur », bien avant le tourbillon de l'IBM PC des années 1980.

ABSTRACT. The article presents the history of an emblematic microcomputer, the Micral system, invented within the R2E company in the 1970s. It aims to show the interplay between levels of technical culture throughout this very complex innovation process: the omnipresence of technological skills, the importance of thinking about uses and users; and the reflexivity of the actors during the creation process - including echoes to the "small is beautiful" trend in the 1970s. Analysis at these various levels makes it possible to give meaning to the conception of Micral and its successive versions: in the 1970s, in a computer world dominated - in industrial terms and in terms of collective representations - by "mainframe" computers, Micral was invented and promulgated the very concept of the "microcomputer", long before the whirlwind of the IBM PC in the 1980s.

MOTS-CLÉS. Innovation, Culture technique, Histoire de la micro-informatique, Micral.

KEYWORDS. Innovation, technical culture, microcomputer history, Micral.

La machine Micral élaborée en 1972-73 s'inscrit dans l'histoire de l'informatique comme étant un des premiers micro-ordinateurs commercialisés ${ }^{1}$. Elle est le produit d'un processus de création et d'innovation complexe, et elle a fait l'objet de contestations en paternité entre deux principaux protagonistes : François Gernelle (le titulaire du brevet en rapport direct avec la machine, déposé en 1973) et André Truong Trong Thi, le directeur de l'entreprise R2E (« Réalisations et Etudes électroniques ») dans laquelle le Micral a été inventé. Un jugement ${ }^{2}$ de 1998 attribue la paternité de l'invention au premier. Mais, d'une part, il est difficile de dissocier dans le processus, le travail de l'ingénieur-concepteur Gernelle, du dirigeant Truong Trong Thi qui a piloté l'entreprise et amené les financements. D'autre part, ramener le processus à deux acteurs est en fait très réducteur, car Micral est le produit d'un collectif pris dans le contexte socio-économique et industriel du tournant des années 1970. L'objet de cet article est de montrer ce qui préside à la genèse du Micral et son évolution, pour donner toute son épaisseur au processus de conception.

Reflet de la complexité du processus, nos analyses reposent sur un ensemble très diversifié et hétérogène de documents et d'archives : manuels techniques, publicités, journaux professionnels, archives conservées au Computer History Museum (CHM / Mountain view, Californie). Le principal

\footnotetext{
${ }^{1}$ Nous renvoyons le lecteur aux ouvrages classiques sur l'histoire de l'informatique, en particulier [CAM 03], [CAM 16], [CER 03] et sur le versant française de cette histoire, on trouvera des éléments généraux dans [LIL 03] et [LAZ 16].

${ }^{2}$ Cours d'appel de Versailles, arrêt n ${ }^{\circ} 951$ du 19 novembre 1998. 
écueil tient à la dispersion et la perte irrémédiable de bons nombres d'archives propres de l'entreprise R2E. Nous avons par ailleurs pu interroger plusieurs acteurs, dont des employés de R2E (François Gernelle, Jean-René Tissot, Bernard Francina, Jean-Marie Ackermann) et Alain Perrier (INRA).

L'analyse de ce processus convoquera plusieurs échelles. Celle du contexte et des cultures techniques en jeu dans ce processus, celle de l'analyse des fonctions et usages du Micral, qui ne cessent d'évoluer, ce qui suppose d'embrasser plusieurs échelles temporelles. Le Micral est en effet conçu initialement comme instrument scientifique et moyen d'automatiser des processus (dans le monde scientifique et industriel). Avant 1974 c'est un ordinateur miniaturisé (plus exactement un miniordinateur miniaturisé), alors que le concept de « micro-ordinateur » s'invente au fil des années 1970, et s'impose progressivement dans l'industrie et dans les esprits, avant de prendre la forme stabilisée et compacte de « clavier-souris-unité centrale-écran » des années 1980.

Précisons encore davantage : un mini-ordinateur correspond à un type de machine inventée dans les années 1960, en rupture avec les grands systèmes «mainframe ». Les minis sont plus compacts, moins chers, mais moins puissants, pour des usages correspondants aux besoins de laboratoires universitaires ou industriels. Le constructeur DEC est le leader du marché dans les premières heures de la miniinformatique. La notion de micro-ordinateur, dans les années 1970, recouvre plusieurs types de machines, ce qui est au cœur de notre problématique : la plupart des micros sont construits autour d'un microprocesseur, mais pas tous ${ }^{3}$; la distinction avec les minis est toujours sujette à caution, nous le verrons, et certains micros peuvent se voir comme des minis miniaturisés (tel Micral à ses débuts) ou bien suffisamment différents pour former une nouvelle catégorie, correspondant à des usages nouveaux. Dans les années 1980, l'avènement de l' «ordinateur personnel ${ }^{4}$ », avec l'IBM PC, fait progressivement coincider micro-ordinateur et ordinateur personnel.

Micral est conçu plus tôt que les autres micro-ordinateurs, et il prend plusieurs formes : selon les versions il s'agit d'un automate, d'un système de contrôle de processus temps-réel, ou d'un microordinateur pour de l'informatique de gestion. Ce pourquoi nous nous référerons au « système » Micral, qui a des propriétés de machine générique (ce qu'on attend d'un ordinateur) et qui est spécialisable à volonté. Micral se distingue des machines produites aux Etats-Unis : on est loin de la «Silicon Valley », des campus universitaires et des clubs de hobbyistes (comme le célèbre « Homebrew computer club ») qui ont fait émerger des innovations et des entreprises qui ont tout emporté sur leur passage (Apple, Microsoft, relayés par l'IBM PC et les microprocesseurs Intel). Ce système Micral montre également que la voie de l'ordinateur personnel (florissante avec l'IBM PC dans les années 1980) n'est pas unique, bien au contraire : R2E-Micral explore dès 1975 de multiples chemins et concepts, intéressants le monde professionnel.

Nos analyses montrent que la génèse du Micral est un processus de création au sens plein du terme, fait de choix et d'opportunités. Nous soulignerons la maîtrise technique des acteurs, leur connaissance étendue de l'électronique qui irriguent les créations successives du système Micral. Nous verrons à quel point ils sont à la fois usagers de la technologie des microprocesseurs (initiée par Intel aux EtatsUnis au tournant de 1970) et dans la capacité de repenser l'intégration de ces machines électroniques dans des systèmes très variés, pour renouveler les usages. Les acteurs du processus ne sont pas pour autant prisonniers de leur savoir-faire : en exerçant une forme de réflexivité sur le processus en train de se faire ils confèrent du sens aux fonctions et usages du Micral.

Pour ces raisons, la notion de culture technique nous paraît utile pour clarifier les enjeux de l'écriture d'une telle biographie, même si une telle notion accepte de nombreuses définitions. Nous suivrons ici [CHO 17], inspiré par [ROQ 83], et notre analyse s'appuyera sur la notion de culture technique telle que ces auteurs la définisse, une notion composite de trois niveaux : le premier niveau

\footnotetext{
${ }^{3}$ L'ordinateur Kenback est un système professionnel mais sans microprocesseur (crée en 1971, donc antérieur au Micral). Il a été vendu à quelques exemplaires seulement [LAZ 16].

${ }^{4}$ Qui peut se caractériser comme un petit ordinateur autonome, économiquement abordable par un individu (ou un petit groupe), utilisable de manière interactive et autonome par un utilisateur (voir des exemples de machines dans [ALL 01] et [ALL 06]). 
relève de la connaissance du fonctionnement des objets, le deuxième englobe les savoir-faire développés pour s'approprier, user des objets et les transformer, le troisième est constitué des savoirs sur le mode d'existence des objets permettant de penser le sens dont ils sont porteurs. Ces distinctions sont éclairantes des enjeux du processus à analyser. Nous avons évoqué dans le paragraphe précédent, la maîtrise technique des acteurs de R2E-Micral, omniprésente et déterminante : cela nous renvoie au premier niveau de la culture technique, qui sera convoquée tout au long de l'article. Les rapports aux usages, si importants dans la conception des Micral correspondent au second niveau. Enfin, derrière la conception du Micral, il nous faut chercher le sens, la manière dont les objets ont été inscrits dans les transformations socio-économiques du moment, et quel rapport nouveau à la technique informatique les acteurs cherchent à instaurer. Micral peut se voir comme un essai de démocratisation de l'informatique, de diffusion d'un instrument " pour tout le monde » (cette portée étant à préciser dans l'article) - c'est ce troisième niveau qui est le plus subtil et difficile à atteindre dans l'analyse.

Pour développer ces analyses, le présent article se construit en trois grandes parties, chronothématiques, qui suivent un itinéraire biographique du système Micral : l'évolution du poids relatif des niveaux de culture technique des acteurs dans le processus d'innovation est donc à suivre tout au long de l'article. La première partie de l'article développe spécifiquement le bain technique et industriel dans lequel émergent la structure R2E et le système Micral, les transformations à tous les niveaux économiques, industriels et culturels qui accompagnent cette émergence de 1970 à 1973. Dans une deuxième partie nous nous focaliserons sur l'invention du Micral lui-même, les choix techniques et économiques, les compromis, les fonctions et les usages ouverts par les concepteurs en 1973. La troisième partie insiste sur les développements du Micral qui devient un micro-ordinateur. L'élaboration du concept même de micro-ordinateur, est d'autant plus problématique qu'il n'existe que des grands ou des «mini » ordinateurs dans les représentations de l'informatique des années 1970. Nous conclurons par un retour sur la complexité de ce processus pour montrer en quoi cette biographie d'innovation peut contribuer aux débats sur la culture technique.

\section{Contexte et cultures techniques : d'Intertechnique à R2E}

Micral est conçu et fabriqué dans la société R2E (Réalisations et Etudes Electroniques), fondée en 1970 dans le secteur de l'électronique industrielle. R2E tire ses premières ressources d'une autre entreprise du même secteur, Intertechnique ${ }^{5}$. Truong Trong Thi, co-fondateur de R2E a été ingénieur pendant 8 ans chez Intertechnique. Gernelle, qui sera au cœur de la conception du Micral, est agent technique et il sera secondé chez R2E par d'autres agents venus d'Intertechnique. Le marché de l'emploi dans le secteur de l'électronique-automatique-informatique, à ce tournant des années 1970, favorise une circulation des hommes, des compétences et des savoir-faire, très fluide à l'échelle francilienne : R2E est géographiquement entourée de nombreuses entreprises françaises ou multinationales (Hewlett-Packard, Varian, Tektronix, etc.).

\subsection{Le bain technique et industriel d'Intertechnique}

Si R2E choisit de se spécialiser dans l'électronique, pour l'instrumentation et l'automatique, Intertechnique a une histoire technique beaucoup plus large ${ }^{6}$. Intertechnique est un équipementier aéronautique fondé en 1951, filiale de la société Dassault. De sous-traitant, Intertechnique devient un groupe international présent sur les marchés des avions civils. Intertechnique participe au développement aéronautique français sur la base d'une stratégie au long cours : assimiler les technologies d'origine américaine (qui sont les leaders du marché) pour ensuite innover à son tour.

A partir de 1956, Intertechnique entame une diversification, sur les marchés du nucléaire, de l'instrumentation et de l'informatique. Au fil des années 1960, en prospectant du côté des Etats-Unis, Intertechnique saisit l'opportunité d'acquérir la maîtrise technologique du mini-ordinateur

\footnotetext{
${ }^{5}$ Après quelques achats et transformations, c'est aujourd'hui le groupe Zodiac-Aerospace.

${ }^{6}$ Pour ce qui touche à Intertechnique nous nous appuyons sur [DAR 93], l'analyse la plus approfondie de l'entreprise à ce jour. 
microprogrammable, porteuse de promesse de transformation dans l'instrumentation [DAR 93]. Intertechnique obtient la licence de fabrication et de vente du fabricant Microdata. Dans le même temps, les commandes changent de nature, sont de plus en plus complexes : au sein d'Intertechnique elles sont gérées par une équipe «Systèmes spéciaux », dont le responsable est Truong Trong Thi. Cet interstice entr'ouvert par Intertechnique est le créneau dans lequel R2E se glissera. Ce créneau est circonscrit par plusieurs éléments : l'approche "sur mesure ", l'automatisation et le contrôle de processus, la mini-informatique qui prend une place toujours plus grande dans ces systèmes.

Tous ces éléments font écho à des transformations de plus grande ampleur en matière électronique et informatique au fil des années 1960-70, lesquelles se manifesteront très clairement dans l'invention du Micral. Le premier mot d'ordre est « miniaturisation » : la course technologique de la Guerre froide, accentuée par la course à l'espace, a été le moteur d'une miniaturisation qui ne cessera pas (le tempo est celui de la Loi de Moore [BRO 06]). Deuxièmement, il faut automatiser les dispositifs industriels, même les plus complexes, pour les accélérer, fiabiliser, rentabiliser. Troisièmement, les systèmes numériques sont en passe de supplanter l'électronique analogique. Enfin, et surtout dans le domaine informatique, les modes de conception évolue vers plus de modularité et de flexibilité dans les usages.

\subsection{La mini-informatique chez Intertechnique}

Pour répondre aux besoins de l'instrumentation et de l'automatisation Intertechnique commercialise des systèmes informatiques à base de mini-ordinateurs. Ces produits s'inscrivent dans le contexte d'une industrie des ordinateurs, à la fin des années 1960, qui est déjà sectorisée en plusieurs catégories de matériels. Les grands systèmes («mainframe ») sont indispensables pour le calcul scientifique et la gestion. Les mini-ordinateurs ont ouvert au fil des années 1960 de nouvelles applications, pour le monde universitaire, les laboratoires de recherche et l'industrie. Les systèmes " temps-réels », importants dans les mondes industriel et militaire, sont au cœur du contrôle de processus. La " bureautique » et les ordinateurs « personnels » émergent tout juste dans les années 1970, et prendront leur envol avec la micro-informatique après 1980.

Concevoir des systèmes industriels complexes est l'apanage de structures solides, comme Intertechnique. Mais avec le microprocesseur, à peine commercialisé, l'horizon va changer très vite : R2E prendra ce créneau car le microprocesseur promet un nouveau mode de conception des objets techniques, avec une grande souplesse et modularité. Dès que le microprocesseur sera une machine suffisamment générique et entièrement programmable (c'est-à-dire à partir de 1972), le centre de gravité des systèmes se déplace du matériel («hardware ») vers le logiciel («software ») : tous les systèmes logiques seront remplacés par du matériel programmable et reprogrammable (autant de fois que nécessaire).

\subsection{Chez Intertechnique ... le "small is beautiful " ne passe pas}

Avant de fonder R2E, Truong Trong Thi pilote des projets d'électronique appliquée à la médecine nucléaire et au traitement du signal, au sein du service "Systèmes spéciaux » d'Intertechnique. Il conçoit des systèmes «sur mesure » dont la demande grandit au fur et à mesure des possibilités des technologies électroniques. Le maître mot est le «temps réel », c'est-à-dire la capacité du système de contrôle à calculer et prendre des décisions sans ralentir le processus. En fonction des projets les compétences d'autres services sont mobilisées (celui de Gernelle notamment) ; la mini-informatique, avec sa puissance de calcul, devient fondamentale et la conception de système intègre des ingrédients matériels et logiciels

L'expérience de Truong Trong Thi chez Intertechnique passe également par les Etats-Unis : il dit lui-même avoir été bouleversé dans ses certitudes et modes d'appréhension de l'électronique à son retour du premier voyage professionnel outre-Atlantique. Dès lors son credo sera de faire toujours plus petit reprenant un mot d'ordre de l'époque : «Small is beautiful ». C'est le titre d'un essai [SCH 73] de l'économiste Ernst Schumacher publié en 1973, critique de la marche de l'économie à l'heure du 
premier choc pétrolier, et insistant sur l' " échelle humaine » (une taille réduite, à l'opposé du gigantisme et de la massification de la consommation), l'idée de capital naturel, le retour à une convivialité (à la Ivan Illich). Le titre est devenu un slogan qui a fait florès, irriguant la culture de l'époque. On peut l'inscrire dans un courant critique post-1968 et environnementaliste. Relativement à Micral, ce « petit » agrège plusieurs sens : le faible encombrement des systèmes électroniques (déjà existants ou à créer) ; la dynamique générale de miniaturisation en cours dans cette industrie, promesse de toujours plus petit ; la promesse d'économie et de réduction des coûts à toutes les échelles, de nouveaux usages (plus conviviaux que les systèmes informatiques gigantesques), de diffusion et d'utilisation pour le plus grand nombre. Micral se comprend uniquement s'il est ré-installé sur ce fond culturel, lequel sera d'ailleurs partagé par toute l'aventure micro-informatique des années 1970, et avec un écho particulièrement important outre-Atlantique.

Cependant ce point de vue de Truong Trong Thi, partagé par Gernelle, n'aura pas les faveurs chez Intertechnique, aux prises avec les systèmes industriels lourds, qui se suffit de la mini-informatique, un marché alors florissant : c'est une des raisons de la fondation de R2E. Le contexte économique, la phase d'informatisation générale de la société et du monde économique constitue la toile de fond commune à Intertechnique et R2E. Sur les brisées d'Intertechnique, R2E s'est développée loin des racines aéronautiques, pour devenir une entreprise d'électronique, spécialisée dans l'instrumentation et les automatismes. Avant même l'invention du Micral, on saisit les échos entre la culture de l'époque et les ambitions de l'équipe qui s'organise, et à quel point le troisième niveau de la culture technique, celui d'une réflexivité sur l'évolution technologique et son inscription en société, joue dans les choix des acteurs de cette histoire.

\subsection{R2E- Réalisations et études électroniques}

L'acronyme R2E indique une volonté de se constituer en "bureau d'études », répondant à des demandes pour systèmes spéciaux et sur-mesure. L'expérience d'Intertechnique prime mais elle évolue au gré des choix de l'équipe R2E, à la fois sur le plan de l'organisation et sur sa volonté d'aller explorer du côté de l'informatique. L'équipe s'identifie d'ailleurs à des " artisans de l'électronique », de l'étude à la réalisation de petites séries.

Suivant l'historiographie, nous avons évoqué deux personnalités centrales dans la conception du Micral chez R2E : A. Truong Trong Thi et F. Gernelle. Truong Trong Thi ${ }^{7}$ est né en 1936 au Vietnam et a fait ses études supérieures en France : il est ingénieur de l'Ecole française de radio-électricitie ${ }^{8}$. Il commence sa carrière en 1959 aux Etablissement Lie Belin (devenu Schlumberger) avant d'entrer chez Intertechnique. Il est un spécialiste de carbotrimétrie (datation par Carbone 14) et a développé un carbotrimètre à transistors en 1961. En fondant R2E en 1970 avec le directeur commercial d'Intertechnique (P. Magneron) il est de ceux qui exfiltrent l'équipe hors d'Intertechnique et embarquent la clientèle associée. Gernelle, avec qui il a déjà une expérience de collaboration, est débauché par R2E en 1971. Jean-René Tissot (né en 1944), issu de l'équipe "Système » d'Intertechnique, rejoindra R2E bientôt en qualité d'ingénieur et responsable technique. La stratégie initiale de R2E est à la fois sur une ligne sur mesure et sur la commercialisation de produits génériques (imprimantes, systèmes d'enregistrement à bande magnétique, à cassettes...). Au sein de la petite équipe qui se structure, l'ingénieur François Gernelle sera l'artisan premier du Micral.

\subsection{Gernelle, une vocation pour l'électronique}

Né en 1944, François Gernelle est un autodidacte de l'électronique, familier de tout ce qui existait dans les années 1960 : modélisme radiocommandé, électronique militaire puis industrie électronique chez Intertechnique. Dans le cadre de son service militaire en 1965-66 il est confronté à l'électronique de Défense, dans une base Radar enterrée. Elle est équipée de calculateurs analogiques à tubes, qui déterminent les trajectoires des avions repérés par Radar, Gernelle est affecté à la maintenance du

\footnotetext{
${ }^{7}$ On trouve une courte biographie, un peu enflammée, de Truong Trong Thi dans [CAH 80]. Truong Trong Thi est décédé en 2005.

${ }^{8}$ Aujourd'hui EFREI (Ecole Française d'Electronique et d'Informatique). 
calculateur $^{9}$ : des armoires de tubes, pour un système de calcul énorme et déjà progressivement supplanté par l'électronique des semi-conducteurs. Il est confronté au gigantisme des installations, la complexité des systèmes informatiques « temps réels » qui assurent l'automatisation d'un réseau de grande ampleur (voir pour plus d'information : [MOU 98], [EDW 96], [RED 00]). Automatisation et temps-réel seront les mots clés de la suite de sa carrière, mais avec une inflexion vers le mini et micro, à l'opposé des installations énormes des années 1960.

En 1968, il entre chez Intertechnique, pour travailler comme agent technique (dans le service « Calculateurs »). Gernelle travaille sur des systèmes d'acquisition de données nucléaires et médicales, aux prises avec les mini-ordinateurs configurés pour le contrôle des processus temps-réel et l'instrumentation. Point singulier dans cette trajectoire, c'est à travers les cours du Cnam diffusés sur les chaines de l'ORTF qu'il se familiarise avec l'informatique d'un point de vue plus théorique [HAY 14]. Il poursuit sur un diplôme d'ingénieur au Cnam, qui le conduira à être débauché par R2E, en 1971, comme ingénieur en électronique.

\section{Inventer le Micral}

Jusqu'en 1972, Gernelle comme Truong Trong Thi sont des utilisateurs des systèmes informatiques. Faire de la «micro-informatique »n'est pas le projet initial de R2E : leur savoir-faire est celui de l'industrie électronique et de l'automatique, étant donnés les hommes, les compétences, les trajectoires qui convergent dans R2E. Le basculement dans la conception de Micral est le résultat d'opportunités et de choix, qui donnent sens à ce processus de conception. Ces choix se trouvent être à la fois en continuité et en rupture avec le monde des minis. Nous sommes à un carrefour décisif pour R2E et la conception de Micral, carrefour auquel se croise la maîtrise technique d'une équipe jeune mais très expérimentée, et le contexte du début des années 1970, avec ses évolutions économiques, industrielles et sociétales. Entrer dans le processus de conception signifie décrire les choix de conception architecturale du premier système Micral.

\subsection{Le microprocesseur au cœur du système}

Micral est un système miniaturisé, qui repose sur une première idée force de R2E : repenser les systèmes électroniques autour du microprocesseur, qui est la grande nouveauté des années 1970, avec l'Intel 4004 puis l'Intel 8008, commercialisé en 1972. Dans son travail de commercialisation du microprocesseur Intel plaide d'ailleurs pour ce recentrage sur une seule puce ${ }^{10}$. Un appel qui sera d'autant plus entendu que les fabricants et les modèles de microprocesseurs vont se multiplier (ils seront près de 30 fabricants en 1975 - [MAL 95]).

Au niveau d'Intel comme de R2E, c'est un pari technique très en phase avec l'ère de miniaturisation que nous avons évoquée, dans un climat de « small is beautiful ». L'Intel 4004 est connu très tôt des professionnels, même si Intel est avant tout un fabricant de mémoires électroniques en semiconducteur. Les services de conception des mini-ordinateurs achètent les mémoires Intel. Utiliser le microprocesseur pour fabriquer des calculatrices et des automatismes est dans l'air du temps. Mais concevoir un ordinateur complet est une autre histoire, celle dans laquelle R2E s'aventure.

\subsection{L'instrumentation bioclimatique : un pari et une opportunité}

Le basculement de R2E dans la conception d'un système complet (le futur Micral) résulte d'une opportunité : R2E répond à une demande d'un client (l'INRA) pour un automate de mesures et de calcul pour l'agronomie. Le choix de R2E est d'y répondre par un système générique programmé pour l'application spécifique demandée d'une part, et de profiter de l'opportunité pour commercialiser la machine générique.

\footnotetext{
${ }^{9}$ Entretien de l'auteur avec F. Gernelle.

${ }^{10}$ En Novembre 1971 la publicité d'Intel pour ses microprocesseurs dans Electronics News l'affiche ainsi : « Announcing a new era of integrated electronics... a microprogrammable computer on a chip ». 
Le contrat passé entre R2E et l'INRA en Juillet 1972 est une forme de pari technique et économique de réaliser une machine plus légère et moins chère que ce qui existe sur le marché. Il s'agit de regrouper des compétences chez R2E pour proposer un système " sur mesure » pour l'INRA, et de rentabiliser en quelque sorte (quasiment) la même équipe et les mêmes compétences sur un projet de petit ordinateur.

Ce pari est porté par une claire vision du potentiel du microprocesseur, en particulier le modèle Intel 8008. C'est la première puce électronique commercialisée dont les spécifications autorisent de faire un système réellement programmable à volonté : Gernelle découvre le «Preliminary Data Sheet » de l'Intel 8008 en 1971 [GER 98]. Au-delà des informations strictement techniques, c'est bien la grande maîtrise technologique de Gernelle qui assure une forme de rationalité analytique ici et porte sa vision des usages possibles du 8008 .

La conception de Micral est une inflexion du côté de R2E, mais aussi du côté de l'INRA. En effet, la culture des agronomes porte plus du côté de la recherche chimique et biologique que du côté des sciences pour l'ingénieur et de l'informatique ${ }^{11}$. L'équipe de bioclimatologie est en fait hors norme, car elle compte des physiciens (rares à l'INRA), qui portent une recherche instrumentale. Entre R2E et l'équipe de bioclimatologie il existe un terrain commun, celui de la conception d'une instrumentation dans toute sa complexité. R2E entrevoit la possibilité de définir une instrumentation non « classique » (comme celle qu'ils élaborent pour le médical et le nucléaire). On trouve des deux côtés un goût pour une recherche-développement exploratoire, une convergence de visions concernant une instrumentation en phase générale d'automatisation.

\subsection{Inventer le Micral - choix technique et développements électroniques}

Le contrat est passé en juillet 1972, et la livraison est assurée en janvier 1973. De l'intention initiale à la concrétisation, à peine 6 mois se sont écoulés. R2E livre donc un système automatisé de collectes de données et de calcul d'évapotranspiration ${ }^{12}$, un système opérationnel, autonome, portable et installable en plein champ, adéquat aux mesures agronomiques. Simultanément l'équipe prépare le dispositif générique (qui sera baptisé « Micral » par la suite), dont l'architecture interne est équivalente. Il est important de saisir ce qui est inventé précisément, le cheminement depuis les premières inspirations.

\subsubsection{Un mini-ordinateur ... miniaturisé}

En 1972, les systèmes mini-informatiques du marché sont susceptibles de répondre techniquement à la problématique de l'INRA, mais ils ont plusieurs défauts : non transportables, chers, avec un environnement de fonctionnement contraignant (refroidissement de la pièce si la machine est confinée) et consommation électrique importante. L'idée de Gernelle, c'est de transposer les multiples cartes électroniques du processeur d'un mini sur une seule carte, avec une puce microprocesseur. L'idée paraît simple mais sa réalisation est extrêmement complexe. C'est tout l'enjeu des 6 mois de conception de 1972 : définir une architecture de machine, faire les choix techniques sur chacun des (nombreux) composants, inventer tous les dispositifs électroniques nécessaires ainsi que les moyens de programmer la machine. Gernelle ne veut pas se contenter de construire un système à microprocesseur pour l'INRA, comme il le dira rétrospectivement : « j'ai évité de " bricoler » comme d'autres personnes le faisaient à l'époque avec le 4004 et le 8008 en utilisant simplement les microprocesseurs pour résoudre une fonction $\gg^{13}$.

\footnotetext{
${ }^{11}$ Entretien de l'auteur avec A. Perrier, qui était alors le responsable de l'équipe.

${ }^{12}$ L'équipe de l'INRA souhaite faire des bilans hydriques relatifs à la culture des plantes : mesurer les quantités d'eau évaporée par le sol et transpirée par les plantes.

${ }^{13}$ Interview de F. Gernelle dans la revue 0/1 informatique ${ }^{\circ}{ }^{\circ 532,} 19$ mars 1979. 


\subsubsection{Technologies micro-électroniques}

Au niveau des composants électroniques, base matérielle de l'ensemble, il faut préciser que R2E ne produit pas de puces électroniques. Les choix de composants répondent à un compromis visant la réduction des coûts dans leur globalité et une fiabilisation du système. D'où leur choix de mémoires en semiconducteur et de circuits logiques consommant un minimum pour obtenir une puissance (électrique) globale de quelques Watt (contrairement aux KWatt dépensés par un mini-ordinateur).

Le système est économe en électricité, plus fiable et portable dans tous les systèmes clients. Le packaging du système, avec sa solidité, assure un fonctionnement dans des conditions beaucoup moins strictes que des mini-ordinateurs.

\subsubsection{Pluribus et «Pile-Canal ${ }^{14}$}

L'architecture du Micral est organisée par une sorte de colonne vertébrale : le Pluribus. Ce bus passif reçoit les cartes selon la destination de la machine à réaliser. Le Pluribus est résolument un choix de conception modulaire : il est doté de 11 connecteurs, pour autant de cartes (processeur, «Pile-Canal », mémoires, entrée/sortie, périphériques).

Au-delà des composants, la problématique de fond dans cette conception est la suivante : le microprocesseur a une vitesse d'exécution limitée ${ }^{15}$, et s'il reçoit sans cesse des informations de l'environnement, il sature. La deuxième idée force de Gernelle est de concevoir un système de mémoire-tampon qui déleste le processeur et régule le traitement des informations. C'est le système de «Pile-Canal » qui servira ensuite à connecter un système de mémoire, de disque ou bande magnétique, et à connecter d'autres ordinateurs au Micral.

A travers ce système on saisit toute l'expérience des systèmes temps-réel acquise au cours des années antérieures, l'importance de la modularité souhaitée, le centrage sur le microprocesseur et les choix architecturaux qui en découlent. Sans la capacité de communication assurée par la Pile-Canal, Micral ne serait pas Micral, et c'est un élément qui le distingue encore plus des « autres » microordinateurs produits à la même époque. La réalisation du système pour l'INRA sera d'ailleurs le premier a bénéficié de cette capacité de communication, multi-canaux.

\subsubsection{Du binaire ... à l’assembleur}

Si les aspects matériels sont la base de la conception, la partie logicielle s'avère tout aussi décisive pour deux raisons fondamentales : d'une part, toute la logique de la machine bascule sur une seule puce programmable ... qu'il faut donc programmer ! D'autre part, le 8008 est une machine presque « nue », Intel ne fournissait aucun logiciel. Tirer parti de la souplesse autorisée par le microprocesseur, c'est tout autant un choix architectural qu'un défi logiciel.

Il faut aussi souligner un point très symbolique : les « grands » industriels de l'informatique se sont acharné à développer des logiciels pour faire fonctionner les grands ordinateurs, depuis les années 1950. L'arrivée sur le marché des machines à base de microprocesseur rappelle à bon nombre d'entre eux une ère «préhistorique » des ordinateurs programmés laborieusement en langage binaire. La micro doit palier à cet inconvénient face à une mini-informatique déjà pleinement développée ${ }^{16}$. Mais une fois passé ce cap logiciel, ce secteur renversera les perspectives de toute l'industrie informatique, au fil des années 1980.

\footnotetext{
${ }^{14}$ Voir le brevet déposé en 1973 : Brevet 2.216.884 «Canal pour l'échange d'informations entre un ordinateur et des organes périphériques rapides », inventeur « François Gernelle ».

${ }^{15}$ Le 8008 fonctionne à une cadence de $500 \mathrm{KHz}$. En termes de calcul, il traite quelques 50000 instructions par seconde. A titre indicatif, un mini-ordinateur comme le PDP8 en traite 350000 ; un ordinateur PC du commerce actuel en traite 100 millions par seconde.

${ }^{16}$ Tout à fait emblématique de la situation, une revue du marché de la mini-informatique en 1974 indique que les micro-ordinateurs sont «très attrayants par leurs coûts mais sont tout de suite limités en raison de leur manque évident de programmes de base ». (Revue « Automatisme et Informatique industrielle », Janvier 1974, p. 15). 
Revenons à R2E : étant donné le degré zéro de systèmes logiciels disponibles, l'équipe va devoir tout construire. C'est le mot d'ordre de Gernelle, pour le hardware comme le software : « lorsqu'on est un jeune ingénieur persuadé d'avoir trouvé une voie royale, et qu'il manque quelque chose, on ne perd pas de temps, on le développe » [GER 98].

\subsection{Bilan : une nouvelle perspective, rompre avec les mini-ordinateurs}

En janvier 1973, la commande de l'INRA est honorée et en avril 1973, la configuration baptisée « Micral » est industrialisée puis commercialisée. Les deux concepts clé du Micral, la structure et la pilecanal sont brevetés dès Février 1973, au nom de Gernelle (pour l'entreprise R2E). Le prototype pour l'INRA est « intégré » dans un boitier propre, mais le Micral autonome est déjà prévu dans sa propre coque en fonte, avec son système d'alimentation. Le Micral est vendu à un prix très bas pour l'informatique de l'époque : 8450 francs en $1973^{17}$. Un mini-ordinateur de qualité comme le DEC PDP-8 en vaut alors 45000 francs $^{18}$ : il est quatre fois plus cher, beaucoup plus puissant en termes de calcul... mais l'usage est en fait très différent. Ce « petit » prix est un levier extrêmement important dans la stratégie de R2E, dès lors qu'il s'agit de diffuser Micral le plus largement possible, au sein du monde professionnel.

\section{Le destin du Micral - inventer le micro-ordinateur}

Sur les 5 années (1973-78) qui suivent la conception du premier Micral, R2E n'a de cesse d'innover, dans un processus qui combine un développement ininterrompu en matériels et logiciels, la conception de nouveaux usages, une commercialisation tous azimuts et une massification de la production. Au fil de son développement Micral va investir deux grands domaines :

- le Micral « automate », qui s'insère dans tout système industriel, et qui fait l'essentiel des ventes R2E jusqu'en 1975,

- le Micral «micro-ordinateur de gestion », qui sera le tournant stratégique pris à partir de 1975 et qui change complètement l'horizon de développement de R2E.

La stratégie est le reflet d'un projet en continuelle construction. Le système Micral n'a rien de figé. Il est fait de nombreux choix déterminants et de la capacité de R2E à saisir des opportunités. Au cour de tout ce processus d'innovation se trouve un concept : le micro-ordinateur. Le concept n'existait pas en 1973 et R2E va orchestrer sa stratégie avec ce concept, autour de ce concept. Faire advenir le microordinateur, avec tous les changements que cela suppose du côté des usagers, donne tout son sens au processus, ce que nous allons montrer. Le faire exister suppose d'articuler un travail de conception technique et industriel, un versant commercial et marketing, une volonté de changer les usages, d'abord dans le monde des automatismes, puis celui de la gestion. Tout cela témoigne d'une volonté de donner un accès élargi aux outils de l'informatique, selon d'autres modèles que ceux proposés par les acteurs institués de l'industrie. Promouvoir Micral est synonyme de s'installer en décalage, voire en rupture avec l'existant. Dans cette phase, la conscience des usages du Micral, renvoyant au second niveau de la culture technique, est primordiale pour nos analyses et donner une pleine compréhension du processus d'innovation.

\subsection{Une première rupture par le " bas de gamme »}

La conception modulaire du Micral permet de faire « grandir » le système à la fois en termes matériels et logiciels, pour démultiplier les applications. Sur le plan matériel, l'enjeu est de développer de nouvelles cartes à introduire sur le Pluribus, à la mesure de l'automate à réaliser (il en existe 50 différentes au catalogue vers 1975). En parallèle, le développement logiciel se poursuit pour faciliter la

\footnotetext{
${ }^{17}$ Pour se donner des ordres de grandeur : 8450frcs correspondent à plus de 50000 euros en 2018. C'est aussi le prix d'une voiture luxueuse en 1973.

${ }^{18}$ Et donc environ 280000 euros en 2018. 
programmation, puis à la faveur de l'implémentation de langages de plus haut niveau, la destination du Micral va évoluer.

En 1973 l'équipe de Gernelle perfectionne Micral avec l'Intel 8008-1 et des performances légèrement supérieures ${ }^{19}$ : c'est le Micral-G. Le premier prend le nom de Micral-N (pour « Normal ») à cette occasion. Ces machines génériques sont commercialisées et intégrées dans différents dispositifs, en particulier pour l'automatisation des instruments scientifiques pour un prix défiant toute concurrence.

Le succès se mesure aux ventes (100 la première année) et dans le $24^{\text {eme }} \operatorname{Sicob}^{20}$ organisé en Septembre 1973. Vu des professionnels et de la presse spécialisée, Micral se trouve catégorisé dans les « mini-ordinateurs », un secteur perçu comme très concurrentiel : «C'est notamment le cas de la série Nova de DATA GENERAL pour le haut de gamme, le Micral de R2E pour le bas ${ }^{21}$. Ce parti pris du «bas de gamme » est fondamental dans la stratégie de R2E : faire moins cher, moins puissant, mais plus rapide pour de nouveaux usages. Ces usages potentiels sont de trois ordres : remplacer les automates industriels existants, en particulier ceux dont la logique est câblée et manque de souplesse ; se placer sur le créneau de petits ordinateurs, moins chers, moins puissants (correspondant à un besoin de petites structures ne pouvant pas se permettre l'acquisition de mainframe ou de minis); créer des usages nouveaux, résultant de cet accès nouveau à des ordinateurs vraiment moins chers.

Le terme « micro-ordinateur » est encore très peu employé dans le Sicob ou les revues professionnelles. Il faut déployer une force de frappe commerciale pour promouvoir à la fois les usages que Micral peut faire évoluer (ou remplacer), laisser imaginer les nouveaux usages ...et faire exister ce concept de « micro-ordinateur».

\subsection{4 - année charnière}

L'année 1974 est une année charnière, marquée par une inflexion de la stratégie : la puissance de calcul du Micral est «bas de gamme », mais les usages peuvent être « haut de gamme », à condition de voir et saisir les opportunités. Les choix et les nouveaux développements de R2E s'opèrent alors dans un contexte en évolution très rapide.

\subsubsection{La stratégie R2E : du "bas de gamme » au micro-ordinateur}

Dans le monde des automatismes, un changement de mode de conception s'annonce : une pensée en système et non plus en composants. R2E sait que son équipe compte parmi les rares «spécialistes » qui intègrent les microprocesseurs dans les systèmes. C'est ce qui assure le développement de " Micralspa », un séquenceur à logique programmable, développé en partenariat avec la société Alspa ${ }^{22}$. Les choix techniques, matériels et logiciels, positionnent Micralspa sur le haut de gamme (des automatismes) d'une part, et sur le remplacement de systèmes qui sont surdimensionnés. Reste à convaincre que ce petit système peut effectivement remplacer la puissance des grands automatismes, c'est le défi de la commercialisation auquel fait face R2E de manière générale.

L'offensive de R2E est tous azimuts sur le plan marketing et commercial : la conception des systèmes spéciaux continue, et relayée par la présence au salon Sicob (1974), des encarts publicitaires, des fiches techniques de produits, des articles dans la presse spécialisée. Pour résumer ce « micro-

\footnotetext{
${ }^{19}$ Le Micral-G est assez peu différent sur le fond mais cadencé à $1 \mathrm{MHz}$ et plus de mémoire RAM (16Ko) il est deux fois plus rapide que le N. Il est commercialisé au prix de 10000 francs en 1974.

${ }^{20}$ Sicob (Salon des industries et du commerce de bureau) est un salon annuel de professionnels de la bureautique et de l'informatique, grand rendez-vous des fabricants et utilisateurs. Le premier Sicob remonte à 1950.

${ }_{21}$ Revue «Automatisme », 1973, p. 423.

22 Société qui fait partie du groupe «Techniques, moyens d'automatisme » de la CGE-Alsthom (Revue « Automatisme et Informatique industrielle », Sept 1974).
} 
ordinateur industriel », à la fois dans la continuité de la ligne R2E, et qui se veut en rupture avec le monde des minis, reprenons les mots d'André Raynaud (direction commerciale de R2E) :

« Micral n'est pas un mini-ordinateur à usage général comme il en existe de nombreux sur le marché. C'est un micro-ordinateur. [...] la gestion des entrées/sorties est particulièrement importante. Cette constatation a donné naissance à Micral : puissance des entrées-sorties, faible coût, faible délai de livraison et surtout faible délai de mise en route dû à une programmation simple. » ( Revue « Automatisme et Informatique industrielle », Février 1974, p. 24)

La stratégie affichée par R2E est de plus en plus claire. Le parti pris du «bas de gamme » est gommé (dans son affichage) au profit de nouveaux mots d'ordre : micro-ordinateur industriel, optimal, adaptable, faible coût. Tout se tient car la réduction des coûts est congruente au choix de réduire la puissance de calcul, en misant sur la capacité de communication du Micral ; le système de communication, très souple, et la structure modulaire, permettent de l'adapter à toute situation ; les choix technologiques pour les composants assurent d'un excellent compromis prix/fonction; tous les éléments du système sont optimisés, c'est une obligation du fait de la puissance de calcul réduite.

La stratégie d'ouverture de R2E commence dans la foulée, en direction de la gestion industrielle, pour sortir des automatismes à strictement parler, dans un partenariat avec l'entreprise Gessix-France. Annoncé début 1974, le Gessix 1000 (G1000) est un dérivé du Micral, un ordinateur de bureau pour l'industrie (PME et PMI), auquel sont adjoints des disques souples (floppy disks). Il est doté d'un langage spécifique et déjà dédié à la gestion. Ce produit où tout est intégré dans un bureau assez volumineux est vendu beaucoup plus cher que le Micral de base : 150000 Francs en moyenne, sur un marché beaucoup plus réduit (de l'ordre de 165 ventes en 1974, 190 en 1975).

\subsubsection{Génération 8080}

Après les Micral-N et Micral-G en 1974, le développement prend, sous la houlette de Gernelle, le tournant du microprocesseur qui succède au 8008. Intel présente son 8080 en avril 1974. Il est important car il est annonciateur de microprocesseurs plus puissants, plus rapides, qui vont directement concurrencer les mini-ordinateurs. Grâce aux choix passés, la structure modulaire du Micral intègre la nouvelle carte processeur avec le 8080, pour ensuite assembler des morceaux déjà développés, et obtenir le Micral-S « Super ». Il sort à l'été 1974 et sera présenté au Sicob en septembre 1974.

Pour ce système « $\mathrm{S}$ », les choix sont clairs. Les performances du 8080 ont un effet très direct sur celles du Micral- $\mathrm{S}^{23}$, annoncé six fois plus rapide que ses prédécesseurs : mais R2E fait l'effort, donc le choix, de le commercialiser quasiment au prix du Micral-G. Autre choix capital, assurer la compatibilité sur toute la lignée Micral. Le « $\mathrm{S}$ » peut utiliser les mêmes logiciels que les précédants. C'est un point très important pour la clientèle, qui n'a pas besoin d'apprendre de nouveaux logiciels à chaque sortie de machine. Mais cela sous-entend un effort de développement interne considérable.

\subsection{5 - microprocesseurs et micro-ordinateurs partout}

L'année 1975 est marquée par le développement exponentiel des technologies à microprocesseurs : ce n'est plus une puce électronique confidentielle, elle est train de devenir la puce centrale des systèmes d'automatisation, et bientôt des ordinateurs. Dans l'industrie de l'instrumentation scientifique l'effet sera considérable ${ }^{24}$. Les fonctions des instruments sont démultipliées : élaboration de résultats de mesures, calculs en temps réels, traitement de l'information, mémorisation, programmabilité des

\footnotetext{
${ }^{23}$ Le manuel est disponible en ligne [URL : http://bitsavers.org/pdf/r2e/MICRAL_S_Microcomputer_Handbook_Aug74.pdf consulté le $19 / 06 / 2018]$.

${ }^{24}$ Le constructeur Hewlett-Packard, très important sur ce marché, annonce que $80 \%$ de ses produits en cours d'étude et de développement sont dotés de microprocesseur en 1975 - (Revue « Automatisme et Informatique industrielle », nov 75).
} 
instruments, autocalibrage. L'instrumentation traverse une petite révolution numérique dont Micral a été un maillon, entre 1971 et 1974. R2E poursuit sa trajectoire dans le monde des automatismes, avant de basculer dans le celui de l'informatique industrielle et la gestion, une fois les développements logiciels assurés : le micro-ordinateur prend sa place, sous deux formes, aussi bien comme " microordinateur industriel » que comme « micro-ordinateur de gestion ».

\subsubsection{Développement logiciel pour le 8080}

Le développement logiciel a pris un tour inattendu chez R2E. Le principal artisan du premier Micral côté logiciel, Maurice Benchétrit, est victime d'une crise cardiaque en 1975. L'équipe est progressivement remaniée, Michel Joubert est embauché en mars 1975, Jean-Marie Ackermann en septembre 1975. Connaissances et compétences se renouvellent, ce qui se révèle indispensable car le 8080 soulève des problématiques d'ingénierie logicielle qui nécessitent une expertise informatique très solide. Les nouveaux ont un cursus universitaire en informatique, et une expérience industrielle, principalement dans les mainframes et mini-ordinateurs. Ils apportent un savoir-faire logiciel au goût du jour, et déficient chez R2E. L'entreprise est un bouillon de culture, fruit d'une hybridation des cultures de l'électronique et de l'informatique en marche avancée.

Après les premières versions du Micral-S de 1974, l'équipe logiciel développe un système d'exploitation beaucoup plus complet, premier étage logiciel indispensable pour rendre la machine utilisable au-delà des automates industriels. Le fruit du développement sera le SYSMIC (Système d'exploitation Micral). Comme pour le reste de Micral, il faut «tout » développer, en un temps record, en s'inspirant de l'existant (systèmes pour les mini-ordinateurs), mais avec une contrainte majeure : la taille limitée de la mémoire et la puissance de calcul réduite des micros. Pour élaborer encore le système, il faut pouvoir programmer la machine dans un langage évolué, et c'est le Basic qui sera choisi : c'est un langage accessible, développé d'abord pour l'initiation à la programmation, il deviendra une pierre angulaire chez R2E.

\subsubsection{L'informatique de gestion, un tournant stratégique}

Du côté de R2E l'équipe de Joubert va concevoir le langage BAL, dérivé du Basic et orienté gestion: Business Application Language. BAL sera la clé de voûte des applications en gestion du Micral dans les années suivantes. Avec les premières machines Gessix, puis le BAL et dans un contexte d'informatisation croissante des PME et PMI, la filière de la gestion devient un horizon de développement à la portée de R2E. En combinant l'expertise du matériel, des périphériques (écrans, clavier, imprimantes) et de sa capacité à développer des logiciels, R2E va développer un ensemble micro-informatique calibré pour la gestion en entreprise. L'aboutissement est le Micral-C présenté en Septembre 1977. Autonome, interactif, avec clavier-écran, programmable pour des applications de gestion, économiquement abordable, il correspond à l'archétype du « futur » micro-ordinateur, tel qu'il se généralise dans les années 1980. 


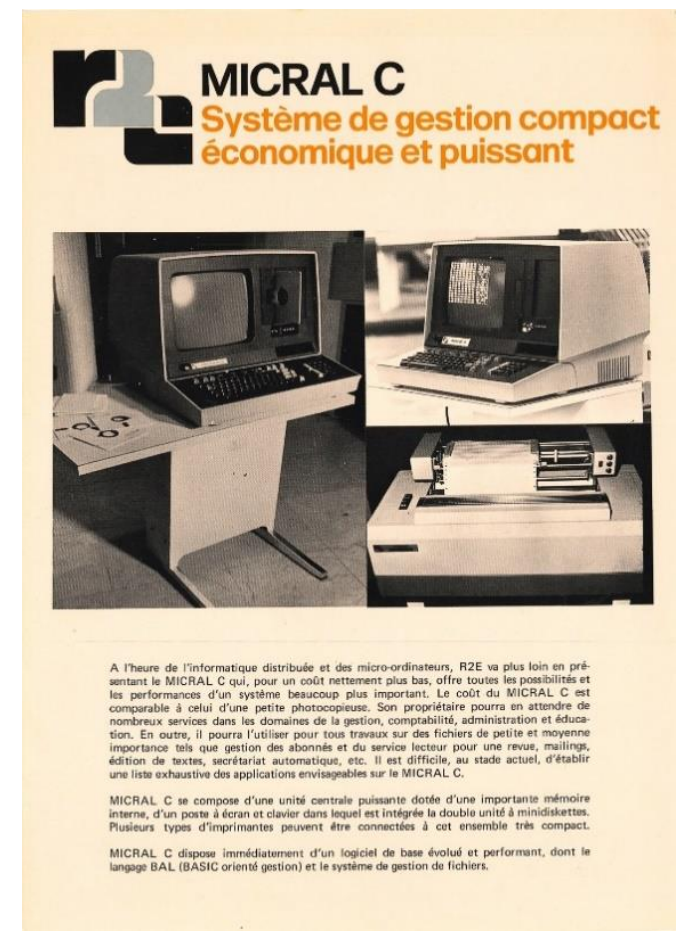

Figure 1. Plaquette de présentation du Micral-C - 1977. Credits Bernard Francina

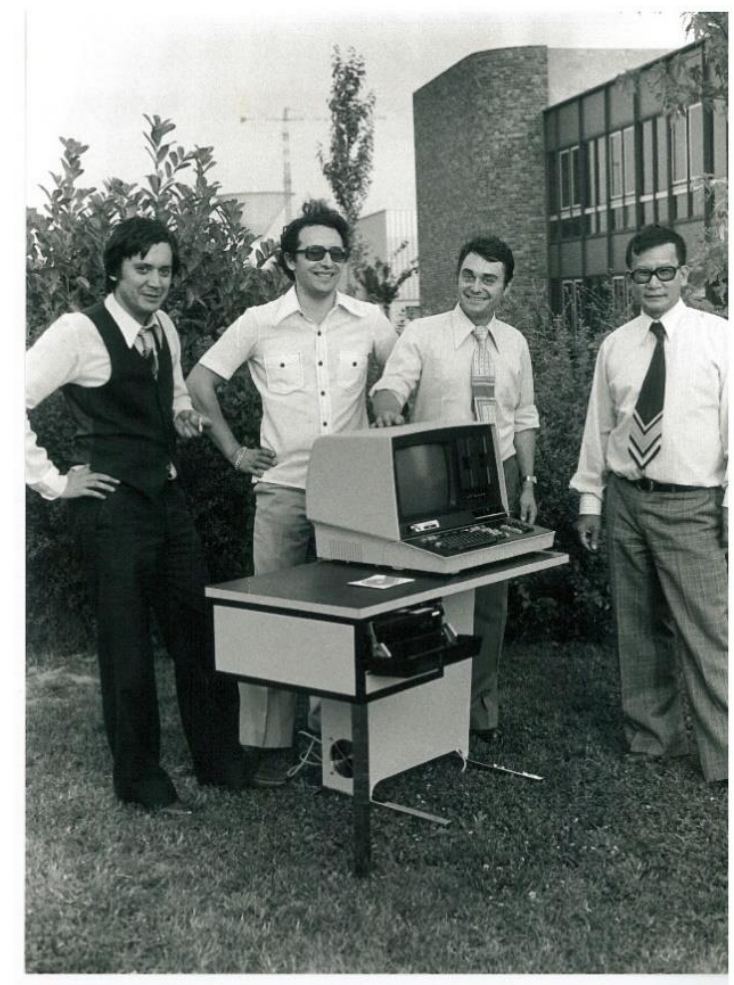

Figure 2. Autour du Micral-C, de gauche à droite : François Gernelle, Bernard Francina, Michel Joubert, André Truong Trong Thi, 1977. Credits Bernard Francina

Tous ces éléments positionneront la micro-informatique R2E à la fois comme rivale de la miniinformatique, et comme complément de la grande informatique. Avec ce tournant de 1975, R2E s'adresse donc à plusieurs types de clients : des grands comptes, qui possèdent une informatique puissante, centralisée, qu'ils souhaitent compléter par une gestion micro-informatique ; des PME et PMI, dont certaines n'ont jamais eu d'ordinateurs - le Micral est leur premier pas dans l'informatique, c'est un point décisif pour R2E ; les automatismes industriels, même si R2E se retire progressivement de ce monde, après 1975. Pour ainsi dire le Micral-S donne une avance considérable à R2E face à tous 
ses concurrents directs ou indirects, français ou américains. Le Micral industriel cohabite avec le Micral de gestion.

Le rapport aux PME/PMI est un aspect capital, parce qu'il correspond à l'identité que R2E veut développer et construire, en investissant le marché de la gestion d'entreprise. Ce n'était pas inscrit à l'avance, et c'était même hors de l'horizon initial de R2E. On saisit le poids et l'importance de cette inflexion de trajectoire en 1975.

Le «small is beautiful » résonne précisément avec cette orientation : faire petit, pour de nouveaux usages... et donner accès au plus grand nombre de professionnels. Une démocratisation en un sens. Mais réservée à un public « sérieux ».

\subsection{Les développements continuent : Micral-M, Micral-V...}

Dans les développements de Micral, R2E mobilise tous ses acquis et maintient ses orientations : assurer la compatibilité des logiciels sur toute sa gamme ; exploiter au maximum les capacités des microprocesseurs ; optimiser les systèmes, pour le meilleur ratio prix / performance ; proposer et développer des usages nouveaux. Le système Micral-M, développé sous la direction de Gernelle, sort en Septembre 1975. Il mise sur l'idée suivante : comme un microprocesseur a une puissance encore modeste, la seule manière d'amplifier la puissance des systèmes est de multiplier les microprocesseurs dans une machine. C'est un système « fédéral ${ }^{25}$.

Les développements portent aussi sur les périphériques qui ont une valeur ajoutée considérable pour les clients. Au catalogue ${ }^{26}$ R2E on trouvera donc des classiques (imprimantes, consoles, claviers), des supports d'enregistrement magnétique, des modules analogiques (cartes multiplexeurs, amplificateurs, convertisseurs), des modules d'entrée / sortie pour l'industrie, etc.

En 1978, R2E possède alors tous les ingrédients matériels et logiciels pour un système d'exploitation temps réel, multiutilisateur et multiposte : force est de constater le grand décalage avec les machines Apple ou les futurs IBM PC, qui vont promouvoir une micro-informatique monotâche, monoposte, « personnelle » pour tout le monde (pour des usages professionnels ou plus familiaux). Enfin, plus anecdotique, mais très symbolique, R2E créé le Micral-V présenté en Septembre 1977 : «Un ordinateur dans une valise ${ }^{27}$. Dans une valise de $55 \mathrm{~cm}$ x $35 \mathrm{~cm}$ x $17 \mathrm{~cm}$ sont assemblés un Micral (à base d'Intel 8080), un lecteur de disquette, un écran et un clavier, une imprimante et l'alimentation. C'est un ordinateur transportable, pour la gestion. On peut le voir comme une apogée des efforts pour donner accès aux machines les plus compactes, au service le plus adapté à l'utilisateur, et aux configurations multiples. Le « small is beautiful » fait machine.

\section{Epilogue et conclusions}

Au fil de ce texte nous sommes entrés dans la complexité du processus qui conduit au Micral, fait de choix innombrables à différentes échelles : du niveau le plus technique jusqu'au niveau marketing, du plus fin compromis matériel aux choix les plus stratégiques pour le développement de R2E. On a insisté sur l'importance de l'assise technologique de ses membres, leur curiosité et leur appétit pour les défis techniques. Au fil des années 1970, la capacité de R2E à créer et inventer est tout à fait exceptionnelle. Micral est un "rêve » d'ingénieurs, pris entre les contraintes techniques du microprocesseur et les opportunités ouvertes par cette puce. C'est ce qui relève d'un second niveau de

\footnotetext{
${ }^{25}$ Le Micral-M est parfois appelé « réseau fédéral » [LIL 75] système fédéral » [RAY 78] - mais il ne semble pas que Gernelle ait luimême choisit ce terme. On parle aussi d' «organisation horizontale » ou de «traitement en parallèle » ou encore de «système distribué ». Micral-M peut embarquer entre 1 et 8 microprocesseurs, chacun étant équipé par des entrées / sorties et des mémoires, interconnecté aux autres.

${ }^{26}$ Catalogues dispersés, mais rassemblé en version anglaise dans (Computer History Museum, document $\mathrm{n}^{\circ} 102665368$ ).

${ }^{27}$ Voir l'article de [RAY 78], le bilan du Sicob 1978 dans « La Revue administrative »; le manuel en version anglaise du Micral-V se trouve en ligne [URL : https://archive.org/details/bitsavers_r2eMICRALVerSystemDec78_3181009 consulté le 16/07/2018]. 
la culture technique, tout à fait essentielle dans le processus de conception. Mais il est impossible de penser tout le processus sans l'inscrire dans une volonté d'ouvrir l'informatique, de démocratiser son utilisation, inspiré par le mot d'ordre "small is beautiful » : au-delà du slogan, le processus traduit une pleine conscience et une réflexivité critique à l'égard de l'informatique, qui se développe au sein d'une sorte de «start-up » en décalage avec la grande industrie informatique, dominante sur les marchés et dans les représentations collectives. La conception du Micral est concomitante de l'élaboration d'une culture technique (celle du troisième niveau) très large parmi les acteurs, qui donne sens au Micral et ses développements. L'itinéraire biographique suivi pour l'analyse du système Micral montre les jeux des différents niveaux de culture technique à l'œuvre parmi les acteurs, à plusieurs moments de ce processus, et contribue à souligner toute l'importance du concept de culture technique comme heuristique des processus d'innovation, de manière plus générale.

Pour ne pas donner une vision trop idéalisée de la biographie du Micral et enrichir encore l'analyse, il faut également indiquer les revers de cette histoire. Les développements chez R2E se poursuivent sans arrêt, mais les difficultés financières s'accumulent entre 1975 et 1978. R2E prospecte pour des partenaires industriels et des investisseurs : sans succès. En 1978, CII-Honeywell-Bull acquiert R2E à 90\%, qui devient la structure R2E-Bull (et lancera des produits étiquetés Bull-Micral). Du bricolage de génie dans une PME innovante à l'industrialisation de micro-ordinateurs il faut franchir un pas énorme, ce que R2E seule n'a pas pu faire. Au sein de R2E-Bull malgré tout, les valeurs de R2E doivent évoluer, se conformer à celle d'une industrie installée, avec sa clientèle. Sur le moyen terme, Gernelle et Truong Trong Thi quitteront la structure, pour de nouvelles aventures industrielles dans le domaine de la micro-informatique, en pleine expansion dans les années 1980 et 1990.

L'histoire de R2E oscille entre continuité, héritage d'un savoir-faire technique et industriel, et volonté de rupture. Micral hérite de la mini-informatique et prépare la rupture micro - à défaut de la réaliser totalement. La transformation sera rapide : en 1977 sort l'Apple II, en 1980 IBM lance son PC... vendu à 1 million d'exemplaire en un an. Les règles du jeu changent, l'échelle de la microinformatique est mondiale, les constructeurs basculeront dans le « compatible PC » pour la plupart (y compris Bull-Micral après 1983). Micro-ordinateur devient pratiquement synonyme d'ordinateur personnel, pour tous les publics (professionnels, familiaux) : on voit la réduction rapide et contrainte à un concept et une trajectoire quasiment unique, alors même que les années 1970 avaient fait émerger plusieurs concepts.

L'autre grande constante, c'est que R2E travaille pour et avec le milieu professionnel, parce que c'est « son » milieu. Il est difficile d'y échapper. Ainsi, les micro-ordinateurs moins « sérieux », ceux pour jouer à la maison ou pour du simple traitement de texte, ne sont jamais entrés vraiment dans l'horizon de R2E, malgré le spectre très large d'usages imaginés : il y a une cloison étanche entre ces mondes, les professionnels du monde industriel dénigrant le moins sérieux. Sur le long terme, ce marché a été sous-estimé par R2E alors qu'il emportera une grande partie de l'industrie après le PC. Tout ceci est à resituer dans un contexte industriel français, bien loin des communautés amateures du type "Homebrew computer club ${ }^{28}$ : il n'y a pas de communauté qui tire profit de Micral, seulement des relations entreprise-clients. Les communautés s'appuient sur une autre culture technique, sont plus radicales dans leur volonté de démocratisation, parfois même utopiques dans leur projet sociotechnique. Outre-Atlantique, la machine Altair commercialisée en 1974 ne disposera pas de programmes "clé en main ": toute l'histoire de son développement et son succès résulte de l'appropriation de ces machines nues par la communauté. Parmi eux, se trouvera Bill Gates qui conçoit une version du langage Basic pour Altair, démarrant l'entreprise Microsoft avec Paul Allen. Preuve de la complexité des rapports symboliques et économiques dans la micro-informatique en émergence, Bill Gates a choisi la rupture et l'affrontement avec le milieu des amateurs pour lancer pleinement Microsoft en 1976, seul moyen sérieux à ses yeux pour se développer en industrie. Au final, au cours des années 1980, tous ces projets communautaires seront dépassés par l'industrie logicielle et la micro-

\footnotetext{
${ }^{28}$ Voir les ouvrages classiques : [CAM 03] et [CAM 14].
} 
informatique promue par l'IBM PC, reprenant à son compte et dans un sens très différent de l'original, le slogan « small is beautiful».

\section{Bibliographie}

[ALL 01] ALLAN ROY A., A History of the Personal Computer: The People and the Technology, London, Allan Publishing, 2001.

[ALL 06] ALLAN ROY A., A Bibliography of the Personal Computer [electronic Resource] : the Books and Periodical Articles, Allan Publishing, 2006.

[BRO 06] BROCK D. (ed.), Understanding Moore's Law. Four decades of innovation, Philadelphia, Chemical Heritage Foundation, 2006.

[CAH 80] CAHIER M., Les impatients ou la Folie de créer, Paris, R. Laffont, 1980.

[CAM 03] CAMPBELL-KELLY M., From airline reservations to Sonic the Hedgehog: a history of the software industry, Cambridge, Mass., MIT Press, 2003.

[CAM 14] CAMPBELL-KELLY M., ASPRAY W., ENSMENGER N., YOST J.R., Computer: a history of the information machine, Boulder, Westview Press, 2014.

[CER 03] CERUZZI P., A history of modern computing, Cambridge (Mass.), MIT Press, 2003.

[DAR 93] DARRIEULAT O., «Intertechnique : un sous-traitant indépendant », Histoire, économie \& société, vol. 12, no 1, p. 137-163, 1993.

[EDW 96] EDWARDS P. N., The closed world: computers and the politics of discourse in Cold War America, Cambridge (Mass.), MIT Press, 1996.

[GER 98] GERNELLE F., "Communication sur les choix architecturaux et technologiques qui ont présidé à la conception du «Micral» premier micro-ordinateur au monde », Toulouse, CEPADUES, 1998.

[HAY 14] HAYAT S., PETITGIRARD L., « Télé-Cnam : enjeux politiques et dispositifs techniques d'une innovation pédagogique », Cahiers d'histoire du Cnam, vol. 1, no 1, p. 127-140, 2014.

[LAZ 16] LAZARD E., MOUNIER-KUHN P., Histoire illustrée de l'informatique, Les Ullis, EDP Sciences, 2016.

[LIL 75] LILEN H., Du microprocesseur au micro-ordinateur: introduction à la micro-informatique, Paris, France, Éditions Radio, 1975.

[LIL 03] LILEN H., La saga du micro-ordinateur: une invention française, Paris, France, Vuibert, 2003.

[MAL 95] MALONE M.S., The Microprocessor: A Biography, New York, Springer-Verlag, 1995.

[CHO 17] CHOUTEAU M., FOREST, J., NGUYEN C., « Quand la culture d'innovation fait écran à la culture technique », Technologie et innovation, , vol. 2, Culture Technique et Culture d'Innovation, 2017.

[MOU 98] MOUNIER-KUHN P., «Calculateurs électroniques et nouveaux systèmes d'armes: Interactions Armées/ Recherche/ Industrie (1946-1959) » dans La IVe République et les problèmes d'armement, Paris, Centre d'études d'histoire de la Défense - ADDIM, p. 376-405, 1998.

[RAY 78] RAYNAUD A., «Le microprocesseur Micral », Sciences et Avenir, Les ordinateurs et la vie quotidienne, no 24, p. 96-102, 1978.

[RED 00] REDMOND K. C., SMITH T. M., From whirlwind to MITRE: the R\&D story of the SAGE air defense computer, Cambridge (Mass.), MIT Press, 2000.

[ROQ 83] ROQUEPLO P., Penser la technique: pour une démocratie concrète, Paris, France, Éditions du Seuil, 1983.

[SCH 73] SCHUMACHER E., Small is beautiful : a study of economics as if people mattered, London, Blond and Briggs, 1973. 